\title{
BMJ Open Active buildings: modelling physical activity and movement in office buildings. An observational study protocol
}

\author{
Lee Smith, ${ }^{1}$ Marcella Ucci, ${ }^{2}$ Alexi Marmot ${ }^{2}$ Richard Spinney, ${ }^{2}$ Marek Laskowski, ${ }^{2}$ \\ Alexia Sawyer, ${ }^{1}$ Marina Konstantatou, ${ }^{2}$ Mark Hamer, ${ }^{1}$ Gareth Ambler, ${ }^{3}$ \\ Jane Wardle, ${ }^{1}$ Abigail Fisher ${ }^{1}$
}

To cite: Smith L, Ucci M, Marmot A, et al. Active buildings: modelling physical activity and movement in office buildings. An observational study protocol. BMJ Open 2013;3:e004103. doi:10.1136/bmjopen-2013004103

- Prepublication history and additional material for this paper is available online. To view these files please visit the journal online (http://dx.doi.org/10.1136/ bmjopen-2013-004103).

Received 23 September 2013 Accepted 9 October 2013

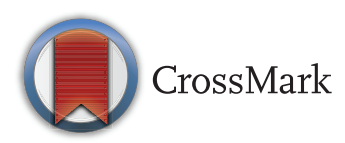

${ }^{1}$ Department of Epidemiology and Public Health, University College London, London, UK ${ }^{2}$ Bartlett School of Graduate Studies, University College London, London, UK ${ }^{3}$ Department of Statistical Science, University College London, London, UK

Correspondence to Dr Lee Smith; lee.smith@ucl.ac.uk

\section{ABSTRACT}

Introduction: Health benefits of regular participation in physical activity are well documented but population levels are low. Office layout, and in particular the number and location of office building destinations (eg, print and meeting rooms), may influence both walking time and characteristics of sitting time. No research to date has focused on the role that the layout of the indoor office environment plays in facilitating or inhibiting step counts and characteristics of sitting time. The primary aim of this study was to investigate associations between office layout and physical activity, as well as sitting time using objective measures.

Methods and analysis: Active buildings is a unique collaboration between public health, built environment and computer science researchers. The study involves objective monitoring complemented by a larger questionnaire arm. UK office buildings will be selected based on a variety of features, including office floor area and number of occupants. Questionnaires will include items on standard demographics, well-being, physical activity behaviour and putative socioecological correlates of workplace physical activity. Based on survey responses, approximately 30 participants will be recruited from each building into the objective monitoring arm. Participants will wear accelerometers (to monitor physical activity and sitting inside and outside the office) and a novel tracking device will be placed in the office (to record participant location) for five consecutive days. Data will be analysed using regression analyses, as well as novel agent-based modelling techniques.

Ethics and dissemination: The results of this study will be disseminated through peer-reviewed publications and scientific presentations. Ethical approval was obtained through the University College London Research Ethics Committee (Reference number 4400/001).

\section{INTRODUCTION}

Increasing population levels of physical activity and reducing sedentary time are major public health priorities in the UK. ${ }^{1}$ Regular physical activity aids in the prevention and management of over 20 chronic conditions, including heart disease, stroke, type 2 diabetes, cancer, obesity, mental health problems and musculoskeletal conditions. ${ }^{2}$ Sedentary time is also associated with negative health outcomes such as an increased risk of metabolic syndrome, independent of physical activity levels ${ }^{3}$ and some data suggest that interruptions in periods of sedentary time are beneficially associated with metabolic health (eg, lower body mass index (BMI), blood glucose and triglyceride levels). ${ }^{45}$

As a result of the growing awareness of the health benefits of physical activity and reduced sedentary time, public health recommendations have been established. It is recommended that adults (aged 19-64 years) should participate in moderate intensity activity for $30 \mathrm{~min}$ on at least 5 days a week and all adults should minimise the amount of time being sedentary for extended periods. ${ }^{1}$ Most guidelines endorse the accumulation of physical activity in short bouts throughout the day $^{6}$ (eg, walking from an office to a meeting room or kitchen). Walking offers a feasible opportunity to incorporate physical activity into adults' daily lives, and it has been suggested that the accumulation of 10000 steps/day is comparable to achieving $30 \mathrm{~min}$ of moderate intensity physical activity per day. ${ }^{7}$

Current levels of physical activity in the UK population are extremely low. In 2008 just $6 \%$ of men and $4 \%$ of women who took part in the Health Survey for England met physical activity guidelines, determined by objective measurement. Estimates of physical activity levels for office-based workers suggest daily step counts of not more than 
$4000-6000 .^{8}$ A previous UK study found a significant difference in meeting physical activity guidelines between different Standard Occupational Classification (SOC) codes in an office environment (managerial, professional and support staff). Male and female managerial workers were $23 \%$ and $17 \%$, respectively, more likely to meet physical activity guidelines than support staff. ${ }^{9}$ However, it is not clear where and when the majority of this physical activity took place (eg, in the office during the day or at home in the evening). The office may offer a platform for activity promotion and reductions in sitting time (one domain of sedentary behaviour), but research in this context is relatively limited.

Two recent reviews showed that physical activity promotion strategies in office buildings can be effective in increasing physical activity levels. ${ }^{10} 11$ The reviews identified that many interventions have been based on bolstering workers' motivation or capability for translating motivation into action, or offering greater physical activity opportunities to those motivated to be more active. However, such interventions have typically produced small effects. They also tend to favour those who are motivated to change and neglect unmotivated workers who may benefit the most. ${ }^{10}$ Some studies have focused on office-based interventions to reduce sitting time, ${ }^{12}$ but a recent systematic review showed that they tended to intervene at only the individual level and were often unsuccessful. ${ }^{12}$ Alternative approaches to increasing workplace physical activity and decreasing workplace sedentary time are needed.

It seems feasible that office layout and, in particular, the distance required to reach key destinations in office buildings (eg, kitchens, print and meeting rooms) could affect both workers' step counts and characteristics of sitting time. In a sample of Australian adults $(n=307)$, those who perceived their office building to have high overall connectivity (eg, hallways and passageways in the office building frequently intersect each other) had significantly fewer breaks in occupational sitting time (b $-0.11 ; 95 \%$ CI -0.17 to -0.06$)$ than those who did not. ${ }^{13}$ The author suggested that this may be partially explained by office workers who perceive their office building to have high connectivity not wanting to leave their desk because this may disturb their colleagues. Significant associations were also found between coworker visibility and coworker proximity and breaks in sitting time. ${ }^{13}$ To our knowledge this is the only observational study to investigate associations between office layout and sitting behaviour, and no work has focused on this association using objective measures. Moreover, the role that the layout of the office may play in facilitating or inhibiting physical activity has yet to be systematically investigated.

Nudging (ie, unobtrusive alterations to social or physical environments to make certain behaviours more likely) populations towards health behaviours is an approach that has been embraced by both the UK and US government, ${ }^{10}$ and could potentially be utilised to increase physical activity levels (eg, step counts) and reduce sitting time. Restructuring office layouts by providing greater physical activity opportunities (eg, manipulating distances to destinations) might nudge workers, whether motivated or not, to increase step counts and thus physical activity levels, as well as to interrupt extended periods of sitting time. Although such interventions would, at best, result in only modest changes in behaviour, any increase in physical activity or reduction in sitting time may improve health, especially if performed on a daily basis. ${ }^{3} 14$ However, before an intervention is carried out, more research is needed to identify and understand associations between office layout (eg, number and distribution of office building destinations), step counts and the characteristics of sitting time.

\section{Aims}

The primary aim of this project was to understand how and where office workers accumulate step counts and spend time sitting in their workplace, to determine the potential for change as a result of spatial reconfiguration of the office layout. This aim will be achieved by testing the hypothesis that features of the office layout (eg, distance to destinations) are associated with step counts and sitting time of office workers. The secondary aim was to identify potential socioecological correlates of occupational physical activity and sitting behaviour, such as environmental perceptions or job role.

This project will use a novel combination of movement assessment technologies along with the application of agent-based modelling (ABM) techniques to inform future interventions on how to maximise step counts and reduce sitting time in office buildings. The ultimate aim was to produce (1) a model of the relationship between spatial reconfiguration of office layout and step counts and sitting time and (2) practical guidance for designers/organisations about features required to create active buildings.

\section{METHODS AND ANALYSIS \\ Project design}

The active buildings project (http://www.activebuildings. co.uk) will be carried out in three phases.

Phase I: Office-based organisations and their employees will be recruited. Data will then be collected on office workers' occupational physical activity, including step counts, and sitting time, as well as potential socioecological correlates of these behaviours.

Phase II: Data collected in phase I will be used to apply $\mathrm{ABM}$ techniques to produce a model of how office buildings could be planned to increase step counts and reduce sitting time.

Phase III: Data collected in phases I and II will be compiled to inform future intervention design.

This paper has a predominant focus on phase I and briefly discusses potential techniques for phase II. Phases II and III will be the subject of future articles. 
Phase I study design

Phase I of the study is cross-sectional in design. Data collection will be administered through two arms: an objective monitoring (ie, accelerometry and location tracking) arm and a questionnaire arm. Both arms will be carried out in 10 buildings (approximately 1.2 buildings per month over 12 months). Within each building, all workers will be asked to complete a questionnaire, and a maximum of 30 workers (to allow for 25 workers after drop out; see sample size calculation) from each building will be recruited to take part in objective monitoring. To maximise data collected and enrich the data on socioecological correlates of workplace physical activity and sitting behaviour (research aim two), only the questionnaire will be administered to all workers in a number of additional buildings (figure 1).

\section{Building inclusion criteria}

UK organisations will be recruited that are within a reasonable travelling distance from the research centre in London. Buildings that the organisations are housed in will be included only if they (1) have a floor area $\geq 2000 \mathrm{~m}^{2}$ (to provide sufficient scale and variation in office layout), (2) house predominantly desk-based workers and (3) have available floor plans.

\section{Participant inclusion and exclusion criteria}

This study will investigate a sample of UK working adults ( $\geq 18$ years). There is no mandatory retirement age in the UK; consequently, no upper age limit has been set for this study. All staff in buildings that are participating in both data collection arms will be invited to complete questionnaires. Based on specific questionnaire responses (ie, desk-based worker and no health condition affecting movement), approximately 30 participants will be recruited from each office building into objective monitoring from the four SOC codes that cover office workers (managers and senior officials, professional occupations, administrative and secretarial occupations and telephone operatives). Although an equal distribution of participants across SOC codes will be sought, this may not be achievable as many office buildings may not house workers across all SOC codes, particularly managers/senior officials and telephone operatives, and some groups may be less likely to take part than other SOC groups owing to time constraints. All staff in buildings that are participating in the questionnaire arm only will be invited to complete questionnaires.

\section{Recruitment}

Potential organisations will be identified through existing links with companies and by engaging companies through presentations at a variety of academic and industry focused events, especially those targeting facility managers or occupational health professionals. After organisations have been recruited, their employees will be recruited using a number of techniques, including: mass emails, posters and presentations, within the organisations.

\section{Procedures}

Data collection procedures will take place over a period of 12 months. Consenting participants will first be asked to complete either a paper or an electronic version of

Figure 1 Study design.




the questionnaire, delivered by the research staff or via email, respectively, at a convenient time.

Next, in the 10 buildings participating in both data collection arms, objective monitoring devices (accelerometer and location tracking device) will be given to a smaller sample of consenting participants, along with verbal and written instructions. At this time, an activity diary/logbook will also be given and anthropometric measurements will be taken. Between 4 and 7 days of accelerometer data are needed to provide a reliable estimate of habitual physical activity. ${ }^{15}$ Thus, participants will be asked to wear objective devices and keep an activity diary for five full days. On day 6 they will return devices and the diary to research staff. The accelerometer will monitor step counts and sitting characteristics through postural allocation (total sitting time; time in prolonged sitting; transitions from sitting to standing/ walking) in and outside the office and the tracking device will only record time-stamped location in the office.

Anthropometric measurements will take place in a private area within the office buildings. Weight will be measured using standard digital bathroom scales. Height will be measured using Leicester height measures. Waist circumference will be measured twice, mid-way between the iliac crest and lower rib using Seca measuring tapes. Blood pressure will be recorded twice using Omron M2 digital blood pressure monitors, after the participant has been sitting at rest for $5 \mathrm{~min}$. Data collection sessions will last approximately $15 \mathrm{~min}$ and will be carried out one-on-one by trained research staff in accordance with standard operating procedure forms.

On return of the objective measuring devices, participants will be interviewed on their preferred routes around the office. Interviews will last approximately $10 \mathrm{~min}$.

\section{Measurement instruments \\ Accelerometer}

Participants taking part in objective monitoring will be asked to wear the ActivPal (http://www.paltech.plus. com; figure 2) all day every day (including during sleep and bathing) for five consecutive days. The ActivPal is a small $(53 \times 35 \times 7 \mathrm{~mm})$, lightweight accelerometer attached to the thigh mid-way between the hip and the knee. In the present study waterproof adhesive tape will be fitted over the device permitting bathing and swimming without the need for removal. The device classifies freeliving activity into periods spent sitting, standing and walking, and it also records step count, cadence and transitions between sitting and standing. The ActivPal has been successfully used in studies of office workers and adults, ${ }^{16}{ }^{17}$ and has been validated for step count, cadence, time spent sitting, standing, walking and for identifying postural transition. ${ }^{18}$ Grant $e t a l^{18}$ found the mean percentage difference between the ActivPal monitor and observation for total time spent sitting, standing and walking was $0.19 \%, 1.4 \%$ and $-2 \%$,

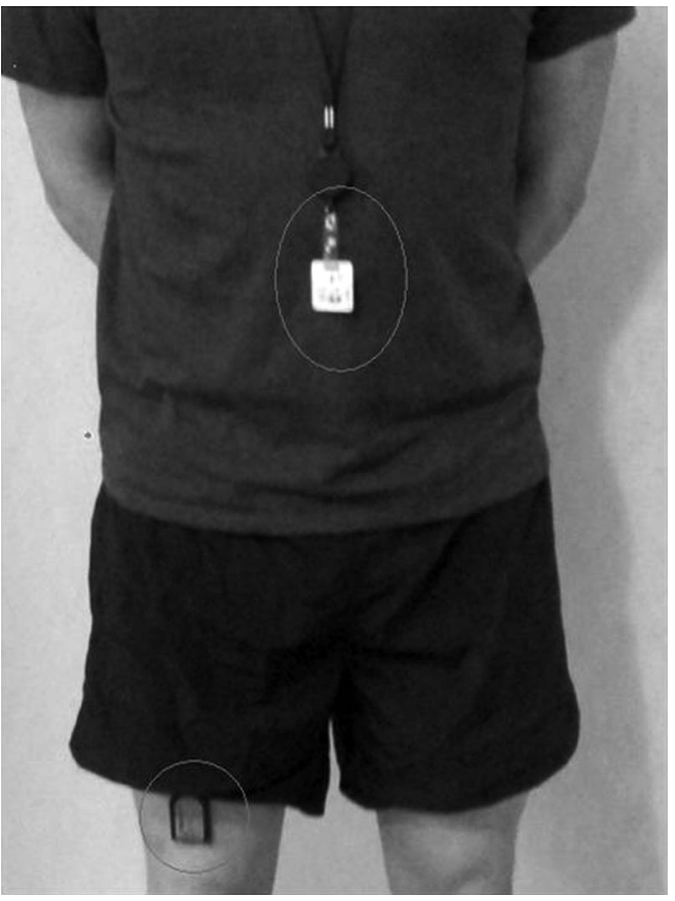

Figure 2 ActivPal accelerometer and radiofrequency identification tracking system to be worn by participants.

respectively. The main advantage of this device in the context of sedentary research is that it employs postural allocation rather than inherently limited methods that use time outside specified count thresholds. ${ }^{19}$

\section{Radiofrequency identification location tracking system}

In addition to the ActivPal, participants in the objective monitoring arm will be given 'OpenBeacon TagPRO' Active Radiofrequency Identification (RFID) badges to be worn on lanyards around the neck (figure 2) every day (but not during sleep or at home) for five consecutive days. These badges are part of the OpenBeacon (http://www.openbeacon.org) sensor system, previously used to study contact patterns between individuals for social $^{20}$ and epidemiological ${ }^{21}{ }^{22}$ research. The badges use low-power radio transmissions to detect proximity between badges as a proxy for close human interaction. Our study extends this method by inferring individuals' interactions with destinations in office buildings (eg, kitchen, print and meeting rooms) by placing badges at the respective destinations. Using this methodology we will infer participants' proximity to the tagged destinations (including participants' desks), in order to ascertain movement patterns within buildings and extract outputs such as the number of trips individuals make from their desk to each destination type. Additional tags along corridors will allow specific routes to be detected. Note that, because of sensitivity to excessive personal data collection or to transmission of data across corporate IT systems, it is anticipated that some participating organisations may be reluctant to permit the RFID location tracking system to be installed. 


\section{Activity diary/logbook}

Participants taking part in objective monitoring will be asked to complete an activity diary/logbook. This is designed to complement the tracking system by gathering information on movement performed during the working day. Activity type and time will be recorded, in addition to information on whether and how participants travelled to other floors in the building. This section of the diary was adapted from the 4-day activity diary used in the Eat Smart study. ${ }^{23}$ It will be completed for 2 days only, minimising the burden on participants.

The diary will also be used to help interpret data from the objective monitoring devices. Additional sections in the diary adapted from logbooks used in the Impact of Constructing Non-motorised Networks and Evaluating Changes in Travel: The Travel to School case study (http://www. iconnect.ac.uk) will record information on times of entering and leaving the office building, days the participant worked away from the office, days they were not working and activities performed while one or both devices were removed. This information will be collected for each day participants wear the objective devices.

\section{Route interviews}

Interviews with participants taking part in objective monitoring will be carried out by trained interviewers. Participants will be shown a floor plan of their office and will be asked to draw the routes they usually take to various office destinations. The interviewer will provide assistance with reading floor plans when needed. The participant will then be asked four questions about the routes they take (1) 'Why did you choose this route rather than taking an alternative route' (2) 'How often do you walk this route in a day' (3) 'What do you like most about this path' and (4) 'What do you like least about this path.' Finally, participants will be asked about multipurpose trips, for example: 'Do you often go to more than one destination within a single trip and if so, why do you combine these destinations.'

\section{Movement at work questionnaire}

All participants taking part in the study will be asked to complete the Movement at Work Questionnaire (see online supplementary additional file 1 ). The questionnaire includes questions on standard demographics, physical activity and sedentary behaviour, as well as potentially important socioecological correlates of workplace activity. Questionnaire completion is estimated to take approximately $10 \mathrm{~min}$.

The Epic Norfolk Physical Activity Questionnaire-2 $\left(\right.$ EPAQ-2) ${ }^{24}$ will be embedded within the Movement at Work Questionnaire to obtain a subjective measure of physical activity levels for all participants. The EPAQ-2 investigates past year physical activity behaviour. It asks questions on three physical activity domains: at work, during leisure time and in the home. Workplace physical activity items were derived from the Modified Tecumseh Occupational Activity questionnaire, ${ }^{25}$ and recreation items were derived from the Minnesota Leisure Time Activity instrument. ${ }^{26}$ Age-adjusted and sex-adjusted correlations between objective measures of daytime energy expenditure and the sum of recreational and occupational reported physical activity (in MET hour/week) in the EPAQ-2 was $0.28(\mathrm{p}<0.001)$, which is largely consistent with correlations between other self-reported and objective measures of activity. The repeatability of the sum of recreational and occupational reported activity was high, $r=0.73 .^{24}$

The Movement at Work Questionnaire also includes items from the Self-Report Habit Index (SRHI) applied to workplace activity behaviour. ${ }^{27}$ The SRHI assesses the repetition and automaticity of behaviour and the extent to which it corresponds with self-identity. This measure has been incorporated to assess whether current workplace activity is carried out automatically, without thinking (ie, habitually). Example questions include 'Is climbing stairs at work something you do automatically' and 'Is climbing stairs at work something you do without consciously having to remember.'

Questions about movement around the office are also in the Movement at Work Questionnaire, for example, 'How many floors do you travel to reach the printer you most often use,' 'Do you usually take the stairs or the elevator' and 'How many times do you usually visit the destination each working day.' Other items in the questionnaire include the 12-item General Health Questionnaire to measures psychological health, ${ }^{28}$ and the Effort-Reward Imbalance Instrument to measure stressful work conditions. ${ }^{29}$ Items to measure perceptions of the physical work environment (eg, my workplace is comfortable/safe from crime/helps me feel creative) are asked, as well as items to measure productivity (eg, doing the following would aid my productivity; going outside at lunch time for a breath of fresh air/ using the stairs instead of the lift whenever I can, etc) and questions on job title, job description and hours worked. Items to capture key demographics (eg, SOC, socioeconomic status, age and sex) and other health-related characteristics (eg, height, weight, history of blood pressure medication and having a health condition that may impair movement) are included.

\section{Spatial metrics}

Spatial variables thought to influence step count and sitting time will be measured in participating buildings taking part in both data collection arms $(\mathrm{n}=10$; figure 1$)$, using floor plans complemented by building audits. Building audits, conducted by trained researchers, will collect general information about the building (eg, location, access to public transport, availability of parking spaces, overall number of floors, age of construction and floor area), and identify the location of different types of office building destinations (eg, meeting room, kitchen, toilet and office area). Office areas will be subdivided into private-enclosed, shared or open-plan, based on classifications similar to those used by Duncan et $_{\text {al. }}{ }^{13}$ 
Floor plans will be used to generate 'axial' and 'segment maps,' concepts developed as part of Space Syntax and Depthmap software, which in turn will be used to calculate distances between each participant's desk and various office building destinations, using methods similar to those described by Sailer and McCulloh. ${ }^{30}$ An axial map is defined as the least set of all longest straight lines covering all parts of the building and minimising depth or steps between spaces. ${ }^{30}$ Lines in an axial map can be considered a way of representing potential routes of movement to and from individuals' workstations, as well as to and from office building destinations. The connection between floors through stairs or elevators is also modelled as a route. A segment map is a refined version of an axial map, where every axial line is broken down into smaller segments at each intersection. Axial and segment maps will be used to calculate four types of distances from each desk to each office building destination (as well as to stairs/elevators): (1) shortest walking distance in metres, (2) fewest steps in an axial map, (3) fewest steps in a segment map and (4) least angle change. These four measurements of distance represent slightly different ways of describing the distance between destinations, in terms of the cognitive, perceived or actual effort involved to overcome those distances. Further details and examples of these metrics can be found in Sailer and McCulloh. ${ }^{30}$ In most cases, distances will be calculated from each desk to its closest office building destination type. In the case of meeting rooms, the distances from each desk to all meeting rooms will be computed and then averaged. If a particular destination type is on a different floor from a desk, then distances including stair and elevator will both be calculated. Proximity of coworkers will also be calculated for each participant, by computing the average distance between the participant's desk to all other desks (1) on their floor and (2) in the entire organisation within that building.

Aside from metrics specific to each participant, overall building metrics will also be calculated from the floor plans, including: floor areas (gross, net internal and net usable) and respective densities (ie, floor area per desk type), as well as floor areas of office building destinations.

\section{Analysis}

Outcomes

The primary outcomes for this study will be average step count and average time spent sitting during office hours, collected by the ActivPal. In addition, the study has been designed to examine the following secondary outcomes, collected by objective measuring devices and participant survey: (1) total overall step counts for the entire wear protocol, (2) total overall sitting (and standing) time for the entire wear protocol, (3) number of transitions made from sitting to standing (breaks in sitting time) during office hours and overall, (4) number of prolonged sitting bouts greater than $1 \mathrm{~h}$ during office hours and overall, (5) total time spent in moderate-to-vigorous physical activity during office hours and overall (min/day), (6) average number of daily trips to each type of office building destination and (7) BMI and other health outcomes (eg, blood pressure).

\section{Quantitative analyses}

To investigate associations between exposures (eg, density of office building destinations; high/low) and outcomes (eg, occupational step count and sitting time) hierarchical regression models will be carried out, adjusted for clustering of outcomes among adults within the same office building, as well as other potential confounding variables (eg, SOC code and ethnicity, etc).

\section{Sample size}

A sample of 250 participants (10 buildings with approximately 25 participants in each) will give $90 \%$ power to detect a difference of $40 \mathrm{~min} /$ day in occupational sitting time at the 5\% significance level using a two-sample $\mathrm{t}$ test between participants in high-office and low-office building destination groups. This is assuming a common SD of $100 \mathrm{~min} /$ day. ${ }^{13}$ This sample size calculation assumes clustering of outcomes among adults within the same office-building is minimal.

\section{ABM techniques}

An exploratory model to understand and predict how office layout influences individual step count as well as sitting time of office workers will be developed. A principal methodology that will be explored will be that of ABM. ABM is a set of approaches used to model complex systems composed of interacting autonomous agents. The use of ABM in physical activity research is relatively novel. $\mathrm{ABM}$ is an alternative approach to the standard multilevel models used in physical activity research ${ }^{31}$ since it 'grows' associations by means of carefully chosen 'bottom-up' rules encoded into the agents' behaviours and interactions. The causal link between exposures and outcomes can then be explored through such mechanisms and can include dynamic processes through which people interact with each other and their environment. This is in contrast to, and in many respects considered as an advancement on, standard multilevel models, since they, like all regression-based approaches, necessarily simplify complex relationships between people and their environment.

The model's structure and methodology will be based on the empirical findings concerning the measured outcomes and their measured correlates from phase I. The model will be designed to explain the findings and predict changes in outcomes by altering various exposure variables such as the number and location of each type of office building destination alongside other attributes such as an individual's likelihood to move. It is anticipated that an agent based approach would consist of numerous agents imbued with attributes such as an 
individualised propensity for movement which would then react to features of the indoor environment and to their colleagues. This would be developed based on behavioural rules on choice of destinations, route finding and so on, derived from subjective and objective evidence gathered during data collection. Detailed background and protocol describing the exploratory model that will be used for the current study will be published elsewhere.

\section{ETHICAL CONSIDERATION AND DISSEMINATION}

Explicit written informed consent will be sought from all study participants. All participants will be informed that they have the right to withdraw from the study at any point without giving reason.

The results of this study will be disseminated to academic audiences through presentations at national and international conferences in physical activity, public health and architecture and through peer-reviewed publications in relevant journals. Results will be disseminated to the public, policy makers, and building designers through seminars and press releases co-ordinated through the UCL Press Office.

Contributors JW, AM, MU, AF, MH and GA designed the active buildings study. All authors contributed to development of the study protocol. LS drafted the manuscript. JW, AM, MU, AF, ML, MH, AS, RS and MK assisted in drafting the manuscript. All authors read and approved the final manuscript.

Funding This work was funded by the National Institute for Health Research's School for Public Health Research (NIHR SPHR). The views expressed are those of the authors and not necessarily those of the NHS, NIHR and the Department of Health.

Competing interests None.

Ethics approval Ethical approval was obtained through the University College London Research Ethics Committee (Reference number 4400/001).

Provenance and peer review Not commissioned; internally peer reviewed.

Open Access This is an Open Access article distributed in accordance with the Creative Commons Attribution Non Commercial (CC BY-NC 3.0) license, which permits others to distribute, remix, adapt, build upon this work noncommercially, and license their derivative works on different terms, provided the original work is properly cited and the use is non-commercial. See: http:// creativecommons.org/licenses/by-nc/3.0/

\section{REFERENCES}

1. CMO. Start Active, Stay Active. 2011.

2. WHO. Global recommendations on physical activity for health. 2010

3. Bertrais S, Beyeme-Ondoua JP, Czernichow S, et al. Sedentary behaviors, physical activity, and metabolic syndrome in middle-aged French subjects. Obes Res 2005;13:936-44.

4. Healy GN, Dunstan DW, Salmon J, et al. Breaks in sedentary timebeneficial associations with metabolic risk. Diabetes Care 2008;31:661-6.

5. Dunstan DW, Kingwell BA, Larsen R, et al. Breaking up prolonged sitting reduces postprandial glucose and insulin responses. Diabetes Care 2012;35:976-83.

6. Haskell WL, Lee IM, Pate RR, et al. Physical activity and public health-updated recommendation for adults from the American
College of Sports Medicine and the American Heart Association. Circulation 2007;116:1081-93.

7. Hatano $Y$. Use of the pedometer for promoting daily walking exercise. ICHPER 1993;29:4-8.

8. Tudor-Locke C, Bassett DR. How many steps/day are enough? Preliminary pedometer indices for public health. Sports Med 2004;34:1-8.

9. Mein GK, Shipley MJ, Hillsdon M, et al. Work, retirement and physical activity: cross-sectional analyses from the Whitehall II study. Eur J Public Health 2005;15:317-22.

10. Abraham C, Graham-Rowe E. Are worksite interventions effective in increasing physical activity? A systematic review and meta-analysis. Health Psychol Rev 2009;3:108-44.

11. Conn VS, Hafdahl AR, Cooper PS, et al. Meta-analysis of workplace physical activity interventions. Am J Preve Med 2009;37:330-9.

12. Chau JY, der Ploeg HP, van Uffelen JG, et al. Are workplace interventions to reduce sitting effective? A systematic review. Prev Med 2010;51:352-6.

13. Duncan MJ, Rashid M, Vandelanotte C, et al. Development and reliability testing of a self-report instrument to measure the office layout as a correlate of occupational sitting. Int $J$ Behav Nutr Phys Act 2013;10:16.

14. Warburton DER, Nicol CW, Bredin SSD. Health benefits of physical activity: the evidence. Can Med Assoc J 2006;174:801-9.

15. Corder K, Brage S, Ekelund U. Accelerometers and pedometers: methodology and clinical application. Curr Opin Clin Nutr 2007;10:597-603.

16. Oliver M, Schofield GM, Badland HM, et al. Utility of accelerometer thresholds for classifying sitting in office workers. Prev Med 2010;51:357-60.

17. Dall PM, Kerr A. Frequency of the sit to stand task: an observational study of free-living adults. Appl Ergon 2010;41:58-61.

18. Grant PM, Ryan CG, Tigbe WW, et al. The validation of a novel activity monitor in the measurement of posture and motion during everyday activities. Br J Sports Med 2006;40:992-7.

19. Kozey-Keadle S, Libertine A, Lyden K, et al. Validation of wearable monitors for assessing sedentary behavior. Med Sci Sports Exerc 2011;43:1561-7.

20. Cattuto C, Van den Broeck W, Barrat A, et al. Dynamics of person-to-person interactions from distributed RFID sensor networks. PLOS ONE 2010;5:e11596.

21. Isella L, Romano M, Barrat $A$, et al. Close encounters in a pediatric ward: measuring face-to-face proximity and mixing patterns with wearable sensors. PLoS ONE 2011;6:e17144.

22. Stehle J, Voirin N, Barrat $A$, et al. High-resolution measurements of face-to-face contact patterns in a primary school. PLOS ONE 2011;6: e23176.

23. Truby $\mathrm{H}$, Baxter KA, Barrett $\mathrm{P}$, et al. The Eat Smart Study: a randomised controlled trial of a reduced carbohydrate versus a low fat diet for weight loss in obese adolescents. BMC Public Health 2010;10:464.

24. Wareham NJ, Jakes RW, Rennie KL, et al. Validity and repeatability of the EPIC-Norfolk physical activity questionnaire. Int $J$ Epidemiol 2002;31:168-74.

25. Ainsworth BE, Jacobs DR Jr, Leon AS, et al. Assessment of the accuracy of physical activity questionnaire occupational data. J Occup Med 1993;35:1017-27.

26. Richardson MT, Leon AS, Jacobs DR, et al. Comprehensive evaluation of the Minnesota Leisure-Time Physical-Activity Questionnaire. J Clin Epidemiol 1994;47:271-81.

27. Gardner B, Abraham C, Lally $P$, et al. Towards parsimony in habit measurement: testing the convergent and predictive validity of an automaticity subscale of the Self-Report Habit Index. Int J Behav Nutr Phys Act 2012;9:102.

28. Hardy GE, Shapiro DA, Haynes CE, et al. Validation of the genera health questionnaire-12 using a sample of employees from England's health care services. Psychol Assess 1999;11:159-65.

29. Siegrist J, Wege N, Puhlhofef F, et al. A short generic measure of work stress in the era of globalization: effort-reward imbalance. Int Arch Occup Environ Health 2009;82:1005-13.

30. Sailer K, McCulloh I. Social networks and spatial configuration-how office layouts drive social interaction. Soc Netw 2012;34:47-58.

31. Smith L, Sahlqvist S, Ogilvie D, et al. Is active travel to non-school destinations associated with physical activity in primary school children? Prev Med 2012;54:224-8. 